\title{
Instability of Optical Solitons in the Boundary Value Problem for a Medium of Finite Extension
}

\author{
VICTOR V. KOZLOV ${ }^{1,2}$ and STEFAN WABNITZ ${ }^{1}$ \\ ${ }^{1}$ Department of Information Engineering, Universita' di Brescia, Via Branze 38, \\ 25123 Brescia, Italy.e-mail: stefano.wabnitz@ing.unibs.it \\ ${ }^{2}$ Department of Physics, St.-Petersburg State University, Petrodvoretz, \\ St.-Petersburg 198504, Russia
}

Received: 7 May 2010 / Revised: 20 August 2010 / Accepted: 7 September 2010

Published online: 23 September 2010 - C) Springer 2010

\begin{abstract}
We consider an integrable nonlinear wave system (anisotropic chiral field model) which exhibits a soliton solution when the Cauchy problem for an infinitely long medium is posed. Whenever the boundary value problem is formulated for the same system but for a medium of finite extension, we reveal that the soliton becomes unstable and the true attractor is a different structure which is called polarization attractor. In contrast to the localized nature of solitons, the polarization attractor occupies the entire length of the medium. By demonstrating the qualitative difference between nonlinear wave propagation in an infinite medium and in a medium of finite extension (with simultaneous change of the initial value problem to the boundary value problem), we would like to point out that solitons may loose their property of being stable attractors. Additionally, our findings show the interest of developing methods of integration for boundary value problems.
\end{abstract}

Mathematics Subject Classification (2010). 37K40 (Soliton theory, asymptotic behavior of solutions), 37C70 (Attractors and repellers, topological structure).

Keywords. soliton, attractors, chiral model, nonlinear optics, boundary-value problem.

\section{Introduction}

Most integrable systems, if not all, are integrable by the method of the inverse scattering problem (ISP). This method allows us to find a number of exact solutions of these nonlinear systems, most known of which are the solitons, by solving the Cauchy problem. When applied to the particular propagation problems in nonlinear optics that are of interest to us here, the Cauchy problem means that some distribution of the optical field is specified at the input boundary (at $z=0$ ) of the semi-infinite medium, and then the evolution of the field is followed during its propagation. A few models in nonlinear optics can be reduced to integrable systems of equations and as a consequence, solutions in the form of optical solitons arise. These solutions are rather important from the physical point of view because as a rule, the solitons present themselves as strong attractors. Whenever the length 
of the medium exceeds the characteristic length which is required for the formation of the soliton, the presence of a (possible) exit boundary at $z=L$ has virtually no influence on the propagation dynamics, and the medium can still be treated as semi-infinite.

More complex situations appear when two optical fields in one-dimensional geometry propagate in opposite directions, that is in a counter-propagating configuration. However even in these cases, under appropriate approximations, the model can be reduced to an integrable system supporting solitons, provided the medium is infinite in both directions, from $z=-\infty$ to $z=+\infty$. In this paper we are dealing with such model (anisotropic chiral field model), which is described by the pair of coupled nonlinear equations [14]

$$
\begin{aligned}
\partial_{\xi} \mathbf{S}^{+} & =\mathbf{S}^{+} \times J \mathbf{S}^{-}, \\
\partial_{\eta} \mathbf{S}^{-} & =\mathbf{S}^{-} \times J \mathbf{S}^{+},
\end{aligned}
$$

governing the evolution of three-dimensional vectors $\mathbf{S}^{+}=\left(S_{1}^{+}, S_{2}^{+}, S_{3}^{+}\right)$and $\mathbf{S}^{-}=$ $\left(S_{1}^{-}, S_{2}^{-}, S_{3}^{-}\right)$along the space coordinate $z$ in the course of time $t$. Here $\xi=$ $(c t+z) / 2$ and $\eta=(c t-z) / 2$ with $c$ as phase speed of light in the medium. Sign " $\times$ " denotes vector product. The coefficient matrix $J$ has specific form

$$
J=\operatorname{diag}(\beta,-\beta,-2 \beta)
$$

with $\beta$ as some constant. Physically, this set of equations describes the situation when two counter-propagating light beams nonlinearly interact inside a spun elliptically birefringent fiber via the Kerr effect. The temporal variation of the beams is supposed to be relatively slow, so that the second and higher order chromatic dispersions can be neglected over the entire length of the fiber. The polarization state of the forward (backward)-propagating beam is described by the Stokes vector $\mathbf{S}^{+}(z, t)\left[\mathbf{S}^{-}(z, t)\right]$. The particular form of the cross-polarization rotation tensor $J$ given by Equation (3) requires the use of a specially manufactured deterministic fiber [without stochastic changes of birefringence along the fiber, see [12] for details and also for a derivation of Equations (1) and (2)]. It may also apply to the case of a randomly birefringent optical fiber, as discussed in [6,13], but in this case the coefficient matrix $J$ has a different form, namely $J=\operatorname{diag}(-\beta, \beta,-\beta)$.

Equations (1) and (2) have solutions in the form of polarization domain soliton

$$
\begin{aligned}
& S_{1}^{ \pm}(z)= \pm \frac{1}{3} \operatorname{sech}\left(\frac{z-z_{0}}{\sqrt{2} L_{n l}}\right), \\
& S_{2}^{ \pm}(z)=\mp \tanh \left(\frac{z-z_{0}}{\sqrt{2} L_{n l}}\right), \\
& S_{3}^{ \pm}(z)= \pm \frac{2}{3} \operatorname{sech}\left(\frac{z-z_{0}}{\sqrt{2} L_{n l}}\right),
\end{aligned}
$$

see [12]. Here $L_{n l}=\beta P$ has the meaning of a nonlinear length. Note that this soliton is a standing-wave solution, i.e., it corresponds to a distribution of the Stokes 
parameters inside the medium which only depends on the propagation coordinate $z$ and does not depend on time. The excitation of this soliton is possible in the case when two counter-propagating beams have equal powers, here denoted as $P$. If the powers of the beams are different, then soliton solutions are also possible, now in the form of moving structures, whose speed linearly depends on the difference of the powers [6,12-14]. These moving solitons are not of interest to us here.

The solitons described by Equations (4)-(6) are configurations of the fields that are frozen inside the infinitely long medium around an arbitrary point $z_{0}$ for an indefinitely long time. Moreover, the solitons are stable against perturbations. The goal of this paper is the analysis of the stability of these solitons in a medium (optical fiber) of finite length $L$.

\section{Polarization Attraction in a Medium of Finite Extension}

First of all, let us identify the main parameters of the model. As a matter of fact there is a single parameter, namely $L_{n l}$, which determines both temporal and spatial scales of the field evolutions. Indeed the characteristic spatial extension of the soliton is equal to $\sqrt{2} L_{n l}$. We also need to choose the length of the medium $L$, which cannot be smaller than $\sqrt{2} L_{n l}$, otherwise the medium cannot fully accommodate the soliton. However, the medium length $L$ cannot also be too large, otherwise the medium is seen as infinite by the soliton. Here we choose a compromise value $L=20 L_{n l}$. Note also that, according to the chosen normalization, the Stokes vectors are normalized in such a way that the power of each beam which is defined as

$$
S_{0}^{ \pm}=\sqrt{\left(S_{1}^{ \pm}\right)^{2}+\left(S_{2}^{ \pm}\right)^{2}+\left(S_{3}^{ \pm}\right)^{2}}
$$

is equal to unity. The equations of motion (1) and (2) imply that power of each beam is a conserved quantity: $S_{0}^{+}(z-c t)=S_{0}^{+}(z=0, t)$ and $S_{0}^{-}(z+c t)=S_{0}^{-}(z=$ $L, t)$ for all $z$. Here we set $S_{0}^{+}(z=0, t)=S_{0}^{-}(z=L, t)=1$ for all $t$, i.e., we consider steady-state boundary conditions.

On the left panel of Figure 1 we show the soliton solution which is constructed according to the formulas (4)-(6). In parallel, we performed numerical simulations based on the method developed in Ref. [5], starting from the boundary

$$
\begin{aligned}
& S_{1}^{+}(z=0, t)=S_{1}^{-}(z=L, t)=0.01, \\
& S_{3}^{+}(z=0, t)=S_{3}^{-}(z=L, t)=0.01, \\
& S_{2}^{+}(z=0, t)=-S_{2}^{-}(z=L, t)=\sqrt{1-0.01^{2}-0.01^{2}}
\end{aligned}
$$

and initial

$$
\begin{aligned}
& S_{1}^{+}(z, t=0)=S_{1}^{-}(z, t=0)=0.01, \\
& S_{3}^{+}(z, t=0)=S_{3}^{-}(z, t=0)=0.01 \\
& S_{2}^{+}(z, t=0)=-S_{2}^{-}(z, t=0)=\sqrt{1-0.01^{2}-0.01^{2}}
\end{aligned}
$$



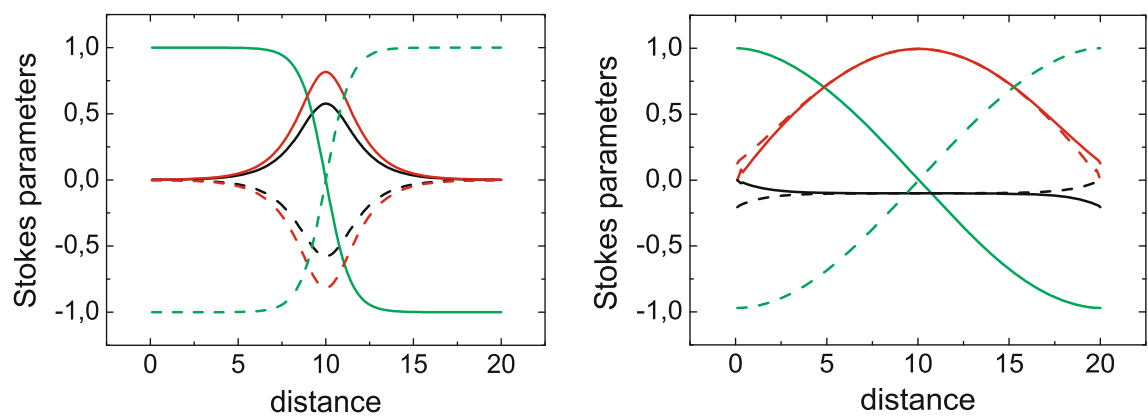

Figure 1. Stokes parameters of forward and backward beams as the function of distance $z$ inside the medium of total length $L=20 L_{n l}$. The shape of the soliton plotted according to Equations (4)-(6) is shown on the left panel. The steady-state solution obtained as a result of the numerical evolution of Equations (1) and (2) with boundary and initial conditions (8)-(13) during a time sufficient for the full convergence to the steady-state regime, is shown on the right panel. Solid (dashed) lines represent Stokes parameters of the forward (backward) beam: $S_{1}^{ \pm}$(red), $S_{2}^{ \pm}$(green), $S_{3}^{ \pm}$(black) (color figure online).

conditions until convergence to a steady-state was observed. The resulting steady-state is shown on the right panel of Figure 1. The steady-state solutions of Equations (1) and (2) are obtained by setting $\partial_{\xi}=-\partial_{\eta}=0.5 \mathrm{~d} / \mathrm{d} z$. The resulting six ordinary differential equations have four invariants, namely, the powers of the two beams $S_{0}^{ \pm}$plus $K=S_{3}^{+}+S_{3}^{-}$and $\Gamma=S_{2}^{+} S_{2}^{-}-S_{1}^{+} S_{1}^{-}+2 S_{3}^{+} S_{3}^{-}$. As a result, the general stationary solutions evolve on a surface resulting from the intersection of these four invariants. In the case of opposite beams with equal power, and guided by the numerical solution shown in the right panel of Figure 1, we may search for those stationary solutions such that $S_{1}^{+}=S_{1}^{-}, S_{2}^{+}=-S_{2}^{-}$, and $S_{3}^{+}=S_{3}^{-}$. In this case, one has $\mathrm{d} S_{3}^{+} / z=0, \mathrm{~d} S_{1}^{+} / \mathrm{d} z=-1.5 S_{2}^{+} S_{3}^{+}$, and $\mathrm{d} S_{2}^{+} / \mathrm{d} z=1.5 S_{1}^{+} S_{3}^{+}$, so that $S_{1}^{+}=A \sin \left(1.5 S_{3}^{+} z+\Phi\right)$, where $A$ and $\Phi$ are determined by the boundary conditions. In the case of Figure 1 the best fit requires $A=-1$ and $\Phi=0$.

By comparing the two graphs on Figure 1 we may first of all point out that the steady-state strongly differs from the soliton. Second, this steady-state solution appears to be a strong attractor and arises from a rather broad class of initial conditions. The time which is necessary for the evolution towards this steady-state sensitively depends on the initial and boundary conditions and quickly grows with the increase of the thickness of the medium $L$. This strong attractor represents a polarization wall. The origin of this name finds its roots in the characteristic shape of $S_{2}^{ \pm}$, which represents a gradual switch of the polarization states of the beams: from $z=0$ to $z=L$ for the forward beam and from $z=L$ to $z=0$ for the backward beam. The spatial evolution of the beams towards the state with polarizations orthogonal to the corresponding inputs is called polarization attraction. Note that the soliton also provides an example of polarization attraction, as it represented by the evolution of $S_{2}^{ \pm}$across the localized soliton profile: see the spatial dynamics of $S_{2}^{ \pm}$on the left panel of Figure 1 . 
In fact, there is a conceptual difference between the soliton and the polarization attractor (as we call the steady-state shown on the right panel of Figure 1). The soliton is a self-localized structure whose width does not depend on the spatial extension of the medium. In contrast, the polarization attractor extends over the entire length of the medium. Therefore the polarization attractor does not appear in an infinitely long medium, as its origin is entirely due to the existence of the two boundaries. In this respect, some dynamical properties of polarization attractors were described in Ref. [7].

Since the polarization attractor does not exist in an infinite medium, in this case the attractor cannot compete with the solitons, and the latter are the stable solutions of wave propagation. The situation changes when the medium becomes finite. The natural question then arises on how solitons and polarization attractors may coexist in a medium of finite extension. Does the soliton keep its stability, at least in some restricted sense? This issue is addressed in the next section.

\section{Formation and Breakdown of Solitons}

Let us consider the same configuration as before but let us apply different initial and boundary conditions, namely,

$$
\begin{aligned}
& S_{2}^{+}(z=0, t)=-S_{2}^{-}(z=L, t)=1.0, \\
& S_{1}^{+}(z=0, t)=S_{1}^{-}(z=L, t)=0, \\
& S_{3}^{+}(z=0, t)=S_{3}^{-}(z=L, t)=0,
\end{aligned}
$$

and

$$
\begin{aligned}
& S_{1}^{ \pm}(z, t=0)= \pm \frac{1}{3} \operatorname{sech}\left[\frac{z-z_{0} \pm z_{\text {shift }}}{\sqrt{2} L_{n l}}\right], \\
& S_{2}^{ \pm}(z, t=0)=\mp \tanh \left[\frac{z-z_{0} \pm z_{\text {shift }}}{\sqrt{2} L_{n l}}\right], \\
& S_{3}^{ \pm}(z, t=0)= \pm \frac{2}{3} \operatorname{sech}\left[\frac{z-z_{0} \pm z_{\text {shift }}}{\sqrt{2} L_{n l}}\right] .
\end{aligned}
$$

Here $z_{0}=10 L_{n l}$ is the center of the medium and $z_{\text {shift }}=L_{n l}$ is a spatial shift, which is applied with different signs to each of the two beams. The initial shapes which are shown in Figure 2 are chosen in such a way that the center of the forward (backward) beam is shifted towards the left (right). Note that the choice of $z_{\text {shift }}=0$ in the above equations corresponds to the soliton solution given by Equations (4)-(6).

The input shapes of Equations (17)-(19) evolve with time inside the medium. The key steps of this evolution are illustrated in Figure 2. After an initial stage, at $T=1000$ the evolution results in the formation of the soliton, whose shape perfectly matches the analytic result given by Equations (4)-(6). For some time this soliton stands still in the center of the medium, but then it starts to slowly move 

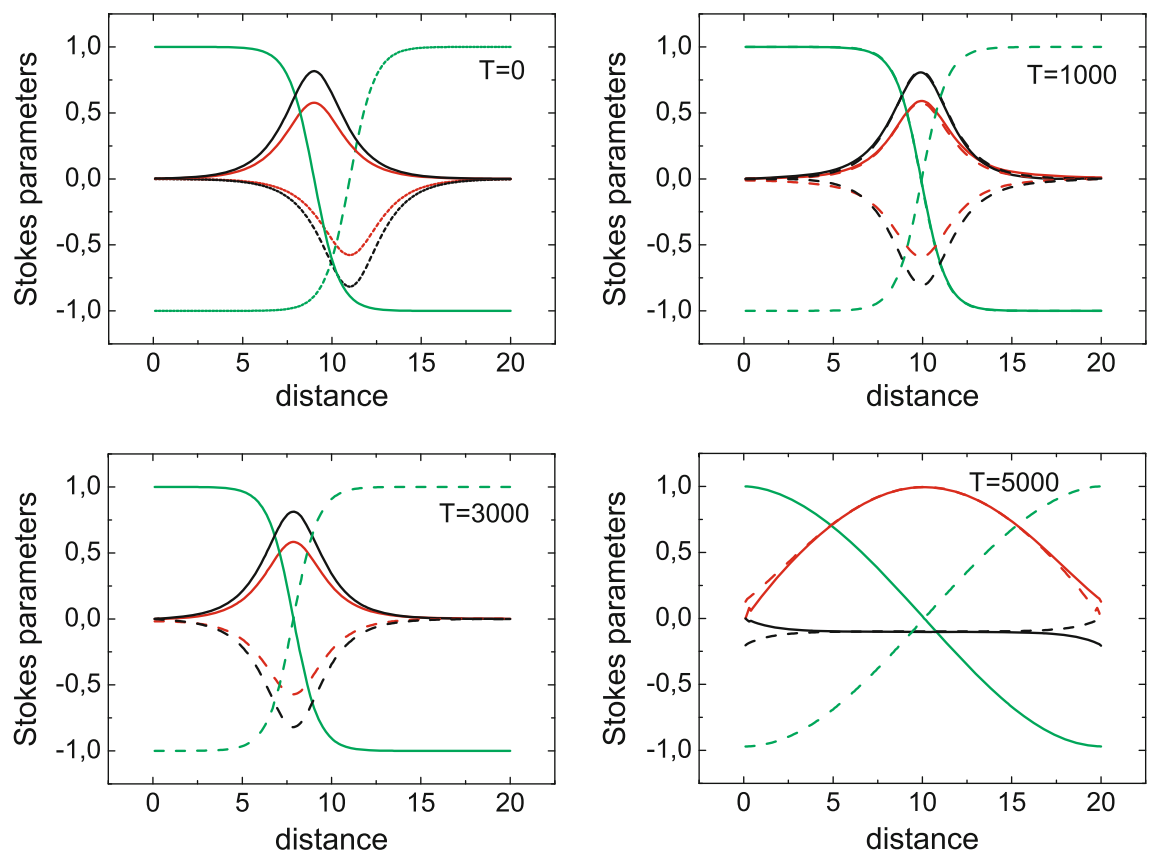

Figure 2. Formation and breakdown of polarization solitons. In all plots we show the Stokes parameters of forward and backward beams as a function of distance inside the medium of total length $L=20 L_{n l}$. Top left input profiles as described by Equations (17)-(19). Top right the result of evolution at $T=1000$, clearly demonstrating the formation of the soliton. The analytical soliton shape given by Equations (4)-(6) is superimposed on the numerically generated shapes. The two sets of the curves are visually indistinguishable. Bottom left snapshot of the evolution at $T=3000$ demonstrating the motion of the soliton towards the left boundary. Bottom right shapes at $T=5000$ demonstrating the ultimate result of the evolution towards the steady-state in the form of the polarization attractor. Comparison of this shape and the shape presented in Figure 1 shows that the ultimate steady-state is the same, although the initial and boundary conditions are different in the two cases. The curves are designated as in Figure 1. Time $T$ is measured in units $L_{n l} / c$. Note that the round-trip time between the boundaries is $T=40$.

towards the left boundary, as demonstrated in the next panel of Figure 2 for $T=$ 3000. Finally, the soliton hits the left boundary and breaks down. After the breakdown of the soliton, the evolution is directed towards the formation of the polarization attractor, as demonstrated in the fourth panel of Figure 2 for $T=5000$. As the steady-state is reached, the field evolution does not change with time anymore.

The conclusion is obvious - the soliton is unstable in a medium of finite length. What is the reason for this instability? It is the asymmetry of the location of the center of the soliton with respect to the middle of the medium. Such asymmetry is not the result of asymmetric initial and boundary conditions, in fact they are chosen to be symmetric, but the result of spontaneously generated (tiny) errors in the course of the numerical simulations. The asymmetry means that right and left wings of the soliton "feel" slightly different boundary conditions. The soliton 


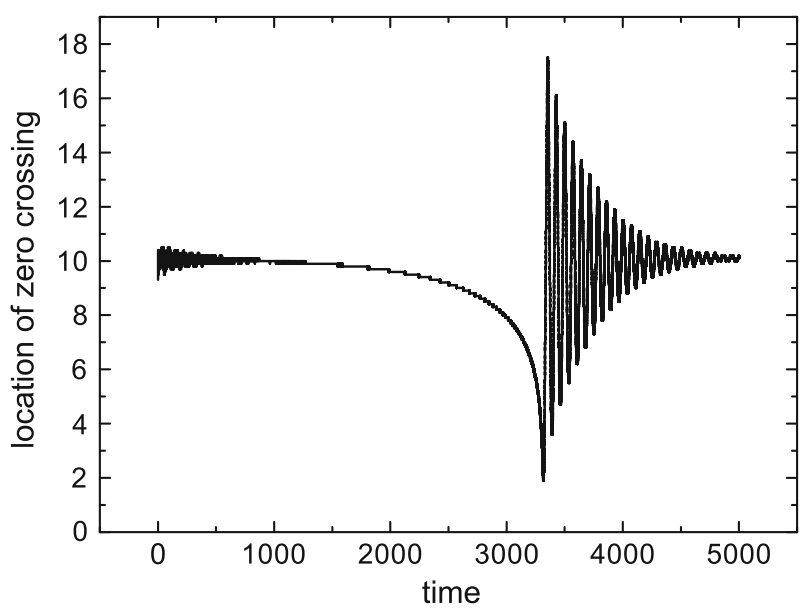

Figure 3. Position of the zero-crossing point (i.e., the point where $S_{2}^{+}=0$ ) inside the medium, as a function of time. Time $T$ is measured in units $L_{n l} / c$.

starts an accelerating motion towards the closest boundary: the closer the boundary, the faster the speed. This accelerated motion is illustrated in Figure 3, where the location of the zero-crossing of $S_{2}^{+}$(i.e., $S_{2}^{+}=0$ ) is followed as a function of time. Until approximately $T=3200$ the soliton keeps its shape, see also the third panel in Figure 2.

It is necessary to separate the previously described type of instability from the trivial disappearance of a moving soliton at the moment when it hits the boundary of a finite medium. Such moving solitons can be realized in the present model by colliding beams with nonequal powers, as noted earlier. However, for the moving solitons the effect of disappearance at the boundary is not the result of instability, i.e., it is not driven by noise. Moreover, the soliton motion itself is present even in an infinite medium and is not caused by the presence of the boundary. In contrast, the motion of the standing-wave solitons which we considered here is not among their intrinsic properties (quite to the opposite, their intrinsic property is to stand still). The motion is solely induced by the presence of the boundary, i.e., it is entirely a boundary effect, which we may term as "boundary attraction."

Indeed, additional simulations (which are not presented here) demonstrated that a same boundary attraction effect is also present if the infinite medium soliton of Equations (4)-(6) is initially placed at a point which is different from the mid-point of the medium; noise is not necessary in this case. Although noise is not needed in this case, nevertheless we deal with the same soliton instability effect, because the slight asymmetry of the wings causes the soliton to move away from its original stable location. Certainly, the farther the boundary, the weaker the effect. The fact that the boundary-induced motion of the soliton does not destroy its shape (see third panel in Figure 2) allows us to conclude that the standing-wave solitons are metastable, that is they have finite life-time. In many 
physical situations metastability is as good as perfect stability, since the observation time is always limited.

The same boundary attraction effect that we have just described also modifies the motion of moving solitons (when the two beams have different powers). However, unless the difference in powers is very small, the boundary-induced motion is too slow to be detectable on the background of the relatively fast intrinsic motion of the soliton.

\section{Conclusions}

We have shown that standing-wave solitons found as solutions of Equations (1) and (2) for an infinite medium are metastable in a medium of finite length. We have reported the attraction effect which is caused by the presence of a boundary. This effect causes an accelerated motion of the soliton until it hits the boundary and disappears. Instead of the soliton, we revealed that the true attractor in the finite medium is the polarization attractor. More information on polarization attractors can be found in Refs. $[1,4,11]$, where the polarization attractor appears as a strong attractor in a model of isotropic fibers. It may be interesting to explore whether mathematical methods and approaches developed there can be accommodated to the treatment of metastability of solitons in the present model.

From the formal viewpoint, we may say that although the asymmetric chiral field model system is completely integrable, such model does not support stable solitons whenever the Cauchy problem is changed to a boundary-value problem. To the best of our knowledge, the boundary-values problems are generally not solvable by means of the inverse scattering transform method, with some exceptions such as Ref. [2]. The present results are of practical relevance for the use of polarization attraction using counter-propagating light beams in optical fibers for implementing a nonlinear polarizer, as it was demonstrated by several recent experiments (see [3] and also Refs. [8-10]).

\section{Acknowledgements}

We thank J. Fatome for sending us a preprint of Ref. [3] prior to its publication. This work was carried out in the frame of the "Scientific Research Project of Relevant National Interest" (PRIN 2008) entitled "Nonlinear cross-polarization interactions in photonic devices and systems" (POLARIZON).

\section{References}

1. Assémat, E., Lagrange, S., Picozzi, A., Jauslin, H.R., Sugny, D.: Complete nonlinear polarization control in an optical fiber system. Opt. Lett. 35, 2025 (2010)

2. Degasperis, A., Manakov, S.V., Santini, P.M.: On the initial-boundary value problems for soliton equations. JETP Lett. 74, 481-485 (2001) 
3. Fatome, J., Pitois, S., Morin, P., Millot, G.: Observation of light-by-light polarization control and stabilization in optical fibre for telecommunication applications. Opt. Express 18, 15311-15317 (2010)

4. Lagrange, S., Sugny, D., Picozzi, A., Jauslin, H.R.: Singular tori as attractors of fourwave-interaction systems. Phys. Rev. E 81, 016202 (2010)

5. Martijn de Sterke, C., Jackson, K.R., Robert, B.D.: Nonlinear coupled-mode equations on a finite interval: a numerical procedure. J. Opt. Soc. Am. 8, 403-412 (1991)

6. Menyuk, C.R., Marks, B.S.: Interaction of polarization mode dispersion and nonlinearity in optical fiber transmission systems. J. Lightwave Technol. 24, 2806-2826 (2006)

7. Pitois, S., Picozzi, A., Millot, G., Jauslin, H.R., Haelterman, M.: Polarization and modal attractors in conservative counterpropagating four-wave interaction. Europhys. Lett. 70, 88-94 (2005)

8. Pitois, S., Millot, G., Wabnitz, S.: Polarization domain wall solitons with counterpropagating laser beams. Phys. Rev. Lett. 81, 1409-1412 (1998)

9. Pitois, S., Millot, G., Wabnitz, S.: Nonlinear polarization dynamics of counterpropagating waves in an isotropic optical fiber: theory and experiments. J. Opt. Soc. Am. B 18, 432-443 (2001)

10. Pitois, S., Fatome, J., Millot, G.: Polarization attraction using counter-propagating waves in optical fiber at telecommunication wavelengths. Opt. Express 16, 6646-6651 (2008)

11. Sugny, D., Picozzi, A., Lagrange, S., Jauslin, H.R.: Role of singular tori in the dynamics of spatiotemporal nonlinear wave systems. Phys. Rev. Lett. 103, 034102 (2009)

12. Wabnitz, S.: Chiral polarization solitons in elliptically birefringent spun optical fibers. Opt. Lett. 34, 908-910 (2009)

13. Wabnitz, S.: Cross polarization modulation domain wall solitons for WDM signals in birefringent optical fibers. Photonic Technol. Lett. 21, 875-877 (2009)

14. Zakharov, V.E., Mikhailov, A.V.: Polarization domains in nonlinear optics. JETP Lett. 45, 349-352 (1987) 